\title{
钯碳/三乙胺体系中邻位三唑取代芳基卤化物的脱卤氢化反应研究
}

\author{
邱会华林白银周鹏* 张建涛刘卫兵 \\ (广东石油化工学院化学学院 广东茂名 525000)
}

\begin{abstract}
摘要 卤代有机物给环境带来了严重的污染, 有机卤代物的脱卤氢化不仅可以将卤代有机物转化为其它化学品, 同时 也可以降低其在环境领域的污染. 以 $\mathrm{Pd} / \mathrm{C}$ 为催化剂, 以三乙胺作为碱、还原剂和氢源, 报道了一种钯催化邻位三坐取 代卤苯脱卤氢化反应. 该方法具有反应体系简单、产物收率高和催化剂可回收等特点.
\end{abstract}

关键词 芳基卤化物; 脱卤氢化; 钯催化; 三唑; 三乙胺

\section{Researches on the Hydrodehalogenation of o-Triazole Aryl Halides in the System of $\mathrm{Pd} / \mathrm{C}$ and $\mathrm{Et}_{3} \mathrm{~N}$}

\author{
Qiu, Huihua $\quad$ Lin, Baiyin $\quad$ Zhou, Peng* Zhang, Jiantao Liu, Weibing \\ (College of Chemistry, Guangdong University of Petrochemical Technology, Maoming, Guangdong 525000)
}

\begin{abstract}
The hydrodehalogenation of halogenated organic compounds which have brought serious pollution to the environment can not only transform them into other usefull chemicals, but also reduce their pollution in the field of environment. A Pd-catalyzed hydrodehalogenation of $o$-triazole aryl halides is introduced, taking $\mathrm{Pd} / \mathrm{C}$ as catalyst and $\mathrm{Et}_{3} \mathrm{~N}$ as base, reductant and hydrogen source. This strategy is highlighted by appealing features such as simple reaction condition, recyclable catalyst, and good yield etc.
\end{abstract}

Keywords aryl halides; hydrodehalogenation; Pd catalyzed; triazole; triethylamine

作为溶剂、药剂、洗涤剂和制冷剂等化工产品的有 效成分，卤代有机物给人类的生产与生活带来了许多便 利. 同时, 此类物质在自然界不断积累, 也给环境带来 了严重的污染, 如氟里昂会加剧温室效应, 多氯代联苯 会污染水土等. 因此, 如何有效实现有机卤代物的脱卤 还原氢化成为有机化学和环境化学领域 ${ }^{[1]}$ 的研究热点之 一.

利用强还原剂如氢气、氢化钠、嗍氢化钠和活泼金 属( $\mathrm{Li}, \mathrm{Ca}, \mathrm{Mg}$ 等)实现芳基卤的脱卤还原, 这些传统的 方法具有较高的活性, 但其反应条件通常比较苛刻, 且 会将一些不饱和基团还原 ${ }^{[2]}$. Buchwald 课题组 ${ }^{[3]}$ 在研究 钯催化芳基溴与仲胺的交叉偶联反应时, 偶然发现了芳 基溴在 $\mathrm{Pd} /$ 磷配体/碱体系中的还原氢化现象. 随后, 基 于类似的钯催化体系，一些还原剂相继应用于芳基卤化 物催化脱卤氢化反应, 如甲酸盐 ${ }^{[4]}$ 、肘 ${ }^{[5]} 、 \mathrm{DMF}^{[6]}$ 、醇和
醇盐 ${ }^{[7]}$ 、多聚甲醛 ${ }^{[8]}$ 、硼氢化合物 ${ }^{[9]}$ 和四甲基二硅氧 烷 ${ }^{[10]}$ 等. 尽管此类方法具有很高的选择性, 但通常需要 配体、腐蚀性无机强碱及较高的反应温度等. 因此, 发 展无额外配体和强碱、催化剂可循环使用的芳基卤的脱 卤还原方法具有非常重要的理论意义和实际应用价值. 作为一种常见的负载型钯催化剂, $\mathrm{Pd} / \mathrm{C}$ 常用于卤代烃的 崖基化反应和交叉偶联反应，具有催化效率高、可循环 利用等特点. 基于其优点, 我们希望发展 $\mathrm{Pd} / \mathrm{C}$ 催化的芳 基卤的脱卤还原新方法.

在研究邻位三唑取代溴苯的环化反应时 ${ }^{[11]}$, 发现底 物在 $\mathrm{Pd} /$ 三乙胺/DMF 体系中会高效率地脱卤还原氢化. 基于此发现，本文将对该反应进行较为系统的研究，并 发展一种分子内三唑配位，三乙胺作为还原剂和氢源的 芳基卤脱卤氢化方法(Scheme 1).

\footnotetext{
* Corresponding author. E-mail: zzd83@aliyun.com

Received December 8, 2019; revised January 4, 2020; published online January 15, 2020.

Project supported by the National Natural Science Foundation of China (No. 21602035), the Natural Science Foundation of Guangdong Province (No. 2016A030307030) and the Program for Innovative Research Team of Guangdong University of Petrochemical Technology (No. 519124).

国家自然科学基金(No. 21602035)、广东省自然科学基金(No. 2016A030307030)和广东石油化工学院(No. 519124)资助项目.
} 


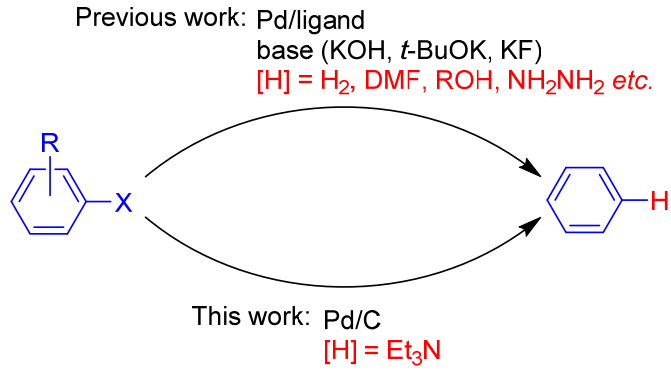

图式 1 钯催化芳基卤的脱卤还原氢化反应

Scheme 1 Pd catalyzed hydrodehalogenation of aryl halides

\section{1 结果与讨论}

\section{1 反应条件优化}

以邻位苄基三唑取代澳苯(1a)为模板底物, 探索底 物脱溴氢化的最佳条件, 考察了钯催化剂、胺和溶剂对 反应的影响, 结果如表 1 所示. 首先, 考察不同钯催化 剂对反应的影响. 以 $\mathrm{PdCl}_{2}$ (5 mol\%) 为催化剂, $\mathrm{Et}_{3} \mathrm{~N}$ (4 $\mathrm{mmol}$ )既作为碱又作为还原剂和氢源, $N, N$-二甲基甲酰 胺(DMF)为溶剂, 于 $100{ }^{\circ} \mathrm{C}$ 反应 $6 \mathrm{~h}$, 发现底物 $1 \mathrm{a}$ 可以 高效地脱溴还原, 以 $94 \%$ 的产率转化为氢化产物 $2 \mathrm{a}$ (表 1 , Entry 1). 当采用其它常见钯盐 $\left[\mathrm{Pd}(\mathrm{OAc})_{2} 、 \mathrm{Pd}\left(\mathrm{PPh}_{3}\right)_{2} \mathrm{Cl}_{2}\right.$ 、 $\mathrm{Pd}\left(\mathrm{PPh}_{3}\right)_{4}$ 作为催化剂时, 反应依然具有很高的效率, 产 率高于 90\%(表 1, Entries 2４). 考虑到负载型钯催化剂 $\mathrm{Pd} / \mathrm{C}$ 的优点, 探索了该催化剂的催化效果, 结果表明反 应产率达到 93\%(表 1, Entry 5). 在无钯催化剂参与的条 件下, 底物 1a 不发生任何反应(表 1, Entry 6). 接下来, 以 $\mathrm{Pd} / \mathrm{C}$ 为催化剂, 考察了不同胺对反应的影响. 当以不 能发生 $\beta-\mathrm{H}$ 消除的 $\mathrm{NPh}_{3}$ 取代 $\mathrm{Et}_{3} \mathrm{~N}$ 时, 反应不发生(表 1, Entry 7); 此外, 当体系没有胺存在时, 反应不发生 (表 1, Entry 10); 实验说明胺在这个反应中扮演了重要 的角色. 当以其它含有 $\beta-\mathrm{H}$ 的胺如 $\mathrm{DABCO}$ 和 $\mathrm{N}(n-$ $\mathrm{C}_{8} \mathrm{H}_{17}$ ) 3 取代 $\mathrm{Et}_{3} \mathrm{~N}$ 时, 产率稍微有所下降(表 1, Entries 8, 9). 最后, 考察了溶剂对反应的影响. 尽管篮选了极性 非质子溶剂(如 DMA 和 DMSO)、极性质子溶剂 (PEG200)和非极性溶剂(对二甲苯), 但它们的效果都不 如 DMF 好(表 1, Entries 11 14). 经过以上实验, 确定反 应的最佳条件为: $\mathrm{Pd} / \mathrm{C}(5 \mathrm{~mol} \%)$ 为催化剂, $\mathrm{Et}_{3} \mathrm{~N}(4$ $\mathrm{mmol}$ )既作为碱又作为还原剂和氢源, $N, N$-二甲基甲酰 胺 $(\mathrm{DMF})$ 为溶剂, 在 $100{ }^{\circ} \mathrm{C}$ 反应 $6 \mathrm{~h}$.

\section{2 反应底物适用范围}

在最佳反应条件下, 对邻位苄基三唑取代溴苯的脱 溴氢化反应的底物适用性进行了研究, 实验结果如表 2 所示. 当底物苄基苯环上连有给电子基团时(如 $4-\mathrm{CH}_{3}$, $4-t-\mathrm{Bu}, 4-\mathrm{CF}_{3} \mathrm{O}$ ), 反应的活性很高, 产率为 $90 \% \sim 94 \%$ (表 2， 2b $\sim 2 \mathbf{d}$ ). 当底物芐基苯环上连有吸电子基团时(如 4-F, 4- $\mathrm{Cl}, 4-\mathrm{NO}_{2}, 4-\mathrm{CF}_{3}, 4-\mathrm{COOCH}_{3}$ ), 反应的产率略有下降,
表 1 反应条件的篮选 ${ }^{a}$

Table 1 Screening of reaction conditions

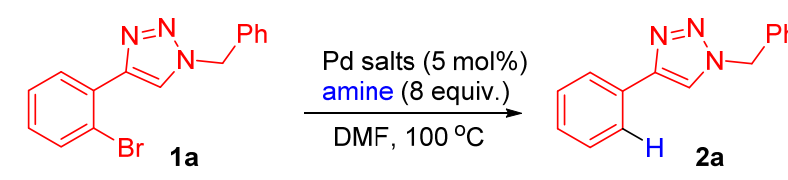

\begin{tabular}{clll}
\hline Entry & \multicolumn{1}{c}{$\begin{array}{c}\mathrm{Pd} \text { source } \\
(5 \mathrm{~mol} \%)\end{array}$} & \multicolumn{1}{c}{ Amine } & Yield $^{b} \%$ \\
\hline 1 & $\mathrm{PdCl}_{2}$ & $\mathrm{Et}_{3} \mathrm{~N}$ & 94 \\
2 & $\mathrm{Pd}(\mathrm{OAc})_{2}$ & $\mathrm{Et}_{3} \mathrm{~N}$ & 92 \\
3 & $\mathrm{Pd}\left(\mathrm{PPh}_{3}\right)_{2} \mathrm{Cl}_{2}$ & $\mathrm{Et}_{3} \mathrm{~N}$ & 90 \\
4 & $\mathrm{Pd}\left(\mathrm{PPh}_{3}\right)_{4}$ & $\mathrm{Et}_{3} \mathrm{~N}$ & 91 \\
5 & $\mathrm{Pd} / \mathrm{C}$ & $\mathrm{Et}_{3} \mathrm{~N}$ & 93 \\
$6^{c}$ & & $\mathrm{Et}_{3} \mathrm{~N}$ & $\mathrm{~N} . \mathrm{R}$. \\
7 & $\mathrm{Pd} / \mathrm{C}$ & $\mathrm{NPh}_{3}$ & $\mathrm{~N} . \mathrm{R}$. \\
8 & $\mathrm{Pd} / \mathrm{C}$ & $\mathrm{DABCO}$ & 89 \\
$9^{d}$ & $\mathrm{Pd} / \mathrm{C}$ & $\mathrm{N}\left(n-\mathrm{C}_{8} \mathrm{H}_{17}\right)_{3}$ & 87 \\
$10^{e}$ & $\mathrm{Pd} / \mathrm{C}$ & & $\mathrm{N} . \mathrm{R}$. \\
$11^{f}$ & $\mathrm{Pd} / \mathrm{C}$ & $\mathrm{Et}_{3} \mathrm{~N}$ & 89 \\
$12^{g}$ & $\mathrm{Pd} / \mathrm{C}$ & $\mathrm{Et}_{3} \mathrm{~N}$ & 41 \\
$13^{h}$ & $\mathrm{Pd} / \mathrm{C}$ & $\mathrm{Et}_{3} \mathrm{~N}$ & 63 \\
$14^{i}$ & $\mathrm{Pd} / \mathrm{C}$ & $\mathrm{Et}_{3} \mathrm{~N}$ & Trace \\
\hline
\end{tabular}

${ }^{a}$ Reaction conditions: $1 \mathrm{a}(0.5 \mathrm{mmol})$, Pd source $(5 \mathrm{~mol} \%)$ and amine (8 equiv.) in DMF $(1.5 \mathrm{~mL})$ at $100{ }^{\circ} \mathrm{C}$ for $6 \mathrm{~h} .{ }^{b}$ Yield of isolated product after column chromatography. ${ }^{c}$ Without Pd salts; N.R is no reaction. ${ }^{d}$ At $120{ }^{\circ} \mathrm{C}$ for $36 \mathrm{~h}$. ${ }^{e}$ Without amine. ${ }^{f}$ Taking DMA as solvent. ${ }^{g}$ Taking DMSO as solvent. ${ }^{h}$ Taking PEG-200 as solvent. ${ }^{i}$ Taking toluene as solvent.

但产率依然较高(表 $2 ， \mathbf{2} \mathbf{e} \sim 2 \mathbf{i}$ ). 当底物芐基苯环的邻位 连有氟取代基时，反应的产率为 $84 \%$ (表 $2,2 \mathrm{j}$ ), 说明苄 基苯环上的位阻对反应影响不大. 当苄基苯环被䒺环取 代时，相应的脱澳氢化反应顺利发生并具有 $89 \%$ 的产率 (表 2, 2k). 接下来, 考察了三唑环氮原子连有烷基的底 物的反应活性. 当氮原子连有 2-苯基乙基或正戍基时, 反应的产率分别为 $88 \%$ 和 $86 \%$ (表 2, $2 \mathbf{I}$ 和 $2 \mathrm{~m}$ ). 当氮原 子连有位置较大的环已基时，反应的时间须延长至 12 $\mathrm{h}$ ，同时反应的产率也有所下降(表 2, 2n). 此外，我们还 考察了溴苯环上电子效应对反应的影响. 实验表明, 澳 苯环上取代基对反应的影响较明显. 当溴苯环上的取代 基 $\mathrm{R}^{2}$ 为推电子基团如甲基和三氟甲氧基时，反应的产 率分别为 $92 \%$ 和 $83 \%$ (表 2, 20 和 $\mathbf{2 q}$ ); 当溴苯环上的取 代基 $\mathrm{R}^{2}$ 为吸电子基团如氟时，反应的产率明显下降(表 2, 2p).

除了上面的底物，还合成了一些 1-(2-溴苯基)-4-(取 代)苯基-1H-1,2,3-三唑，并考察这些底物的脱澳氢化反 应活性. 发现此类底物的反应活性不如前述的邻位苄基 三唑取代溴苯. 将反应温度升高至 $110{ }^{\circ} \mathrm{C}$, 将反应时间 延长至 $24 \mathrm{~h}$, 相应的脱溴氢化反应才转化完全(Scheme 
表 2 三唑导向邻位芳基溴的去溴氢化反应 ${ }^{a, b}$

Table 2 Triazole-directed hydrodehalogenation of aryl halides
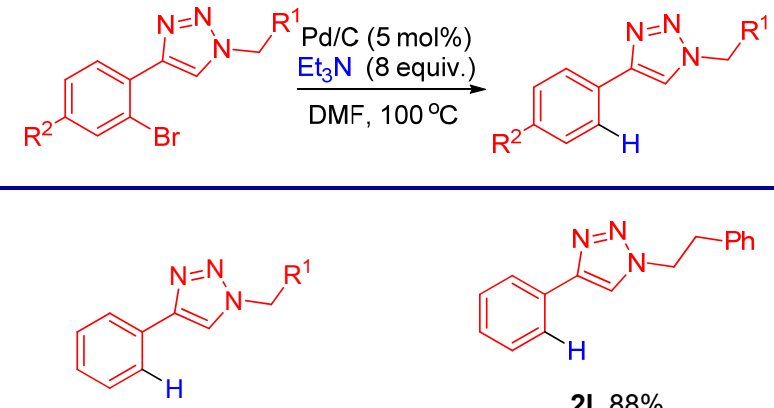

2I, $88 \%$

2a, $\mathrm{R}^{1}=\mathrm{Ph}, 93 \%$

2b, $\mathrm{R}^{1}=4-\mathrm{CH}_{3} \mathrm{C}_{6} \mathrm{H}_{4}, 94 \%$

2c, $\mathrm{R}^{1}=4-t-\mathrm{BuC}_{6} \mathrm{H}_{4}, 93 \%$

2d, $\mathrm{R}^{1}=4-\mathrm{CF}_{3} \mathrm{OC}_{6} \mathrm{H}_{4}, 90 \%$

2e, $\mathrm{R}^{1}=4-\mathrm{FC}_{6} \mathrm{H}_{4}, 88 \%$

2f, $\mathrm{R}^{1}=4-\mathrm{ClC}_{6} \mathrm{H}_{4}, 89 \%$

2g, R $\mathrm{R}^{1}=4-\mathrm{NO}_{2} \mathrm{C}_{6} \mathrm{H}_{4}, 85 \%$

2h, $\mathbf{R}^{1}=4-\mathrm{CF}_{3} \mathrm{C}_{6} \mathrm{H}_{4}, 87 \%$

2i, $\mathrm{R}^{1}=4-\mathrm{CO}_{2} \mathrm{CH}_{3} \mathrm{C}_{6} \mathrm{H}_{4}, 85 \%$

$2 \mathrm{j}, \mathrm{R}^{1}=2-\mathrm{F}-\mathrm{C}_{6} \mathrm{H}_{4}, 84 \%$

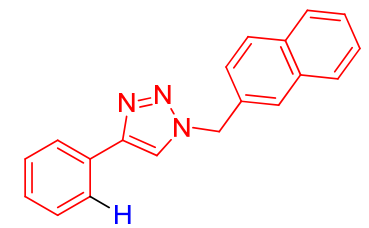

2k, $89 \%$

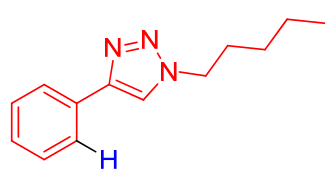

$2 \mathrm{~m}, 86 \%$

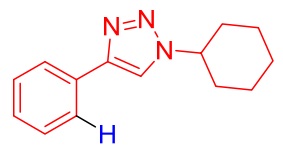

2n, $67 \%^{c}$

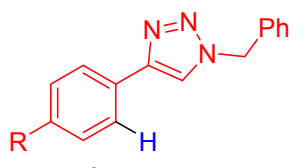

2o, $\mathrm{R}^{2}=\mathrm{CH}_{3}, 92 \%$

$2 p, R^{2}=F, 78 \%$

2q, $\mathrm{R}^{2}=\mathrm{OCF}_{3}, 83 \%$

${ }^{a}$ Reaction conditions: $1(0.5 \mathrm{mmol}), \mathrm{Pd} / \mathrm{C}(5 \mathrm{~mol} \%)$ and $\mathrm{Et}_{3} \mathrm{~N}$ (8 equiv.) in DMF $(1.5 \mathrm{~mL})$ at $100{ }^{\circ} \mathrm{C}$ for $6 \mathrm{~h} .{ }^{b}$ Yield of isolated product after column chromatography. ${ }^{c}$ At $100{ }^{\circ} \mathrm{C}$ for $12 \mathrm{~h}$.

2).
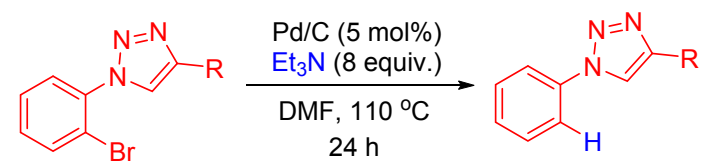

3a, $\mathrm{R}=4-\mathrm{CH}_{3} \mathrm{C}_{6} \mathrm{H}_{4}, 90 \%$

3b, $\mathrm{R}=4-\mathrm{CH}_{3} \mathrm{OC}_{6} \mathrm{H}_{4}, 89 \%$

3c, $\mathrm{R}=n-\mathrm{C}_{4} \mathrm{H}_{9}, 84 \%$

图式 2 1-(2-溴苯基)-4-取代苯基-1H-1,2,3-三唑的去溴氢化反 应

Scheme 2 Hydrodehalogenation of 1-(2-bromophenyl)-4-substituedphenyl-1H-1,2,3-triazole

常见的卤代芳烃包含氟代、氯代、溴代和碘代芳烃， 在此对不同卤代苄基三唑取代卤苯的脱溴氢化反应进 行了比较(Scheme 3). 对于碘代底物, 其脱卤氢化活性 与溴代底物相差不大. 对于氯代底物, 其反应活性急剧 下降，分离产率低于 5\%. 对于氟代底物, 在标准反应条 件下完全不反应.

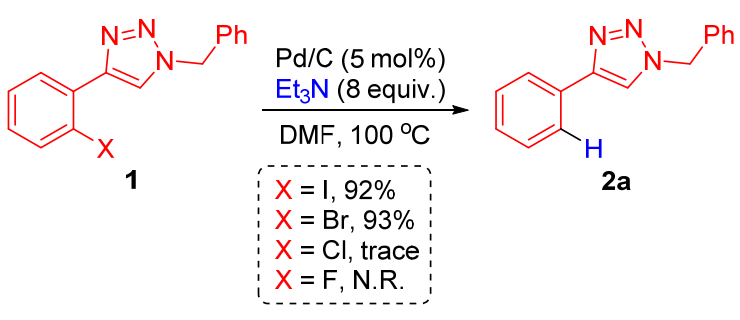

图式 3 邻三唑取代卤苯的脱卤氢化反应活性比较 Scheme 3 Comparison of hydrodehalogenation activities of $o$-triazole substituted halobenzenes

\section{3 放大实验及催化剂重复使用实验}

考虑到 $\mathrm{Pd} / \mathrm{C}$ 催化剂的可循环特点, 以 $2 \mathrm{mmol}$ 的 1a 为底物, 进行了反应的扩大实验和催化剂循环利用实 验. 每次反应结束后, 将反应体系抽滤, 并依次用乙酸 乙酯、乙醇和蒸馏水洗涤滤渣, 然后干燥回收, 再在标 准反应条件下使用回收的催化剂, 考察催化剂的循环催 化性能. 催化剂在第一次使用时, 目标产物的分离收率 为 $83 \%$. 在第二次和第三次循环使用时, 转化效率依次 降低, 分离产率分别为 $67 \%$ 和 51\%. 催化活性下降可能 与催化剂多次使用后活性钯物种的流失有关.

\section{4 反应机理}

针对三乙胺在反应中扮演的角色, 进行了一些控制 性实验(Scheme 4). 标准反应条件下, 以 DMF- $d_{7}$ 取代普 通的 DMF 作为溶剂, 反应得到的产物并没有氛代产物, 说明 DMF 并非氢源. 为了排除溶剂中水的影响, 在标 准反应条件下, 我们往反应体系中加入 $0.1 \mathrm{~mL} \mathrm{D}_{2} \mathrm{O}$ (DMF 和 $\mathrm{Et}_{3} \mathrm{~N}$ 经过无水处理), 反应得到的产物也没有 氛代产物, 说明 $\mathrm{D}_{2} \mathrm{O}$ 也并非氢源. 上述实验可以说明 $\mathrm{Et}_{3} \mathrm{~N}$ 是反应的氢源. 根据实验结果及文献报道 ${ }^{[12]}$, 提出 了可能的反应机理(Scheme 5). 首先, 零价钯与芳基卤 化物发生氧化加成反应, 得到中间体 A. A 与三乙胺反 应脱去一分子澳化氢并得到中间体 B. B 的乙基经过 $\beta-\mathrm{H}$ 消除并实现钯-氮键和钯-碳键的断裂，得到中间体 $\mathbf{C}$. 最后, $\mathbf{C}$ 经过还原消除得到脱溴氢化产物.

\section{2 结论}

报道了一种钯催化邻位三唑取代卤苯脱卤氢化还 原反应，为环境化学领域芳基卤的处理提供了一种新方 法. 在本反应中, 三乙胺既作为碱又作为还原剂和氢源. 该反应体系简单, 产物收率高, 催化剂为可回收的 $\mathrm{Pd} / \mathrm{C}$.

\section{3 实验部分}

\section{1 仪器与试剂}

德国 Bruker 公司 $400 \mathrm{MHz}$ DRX-400 核磁共振仪, 

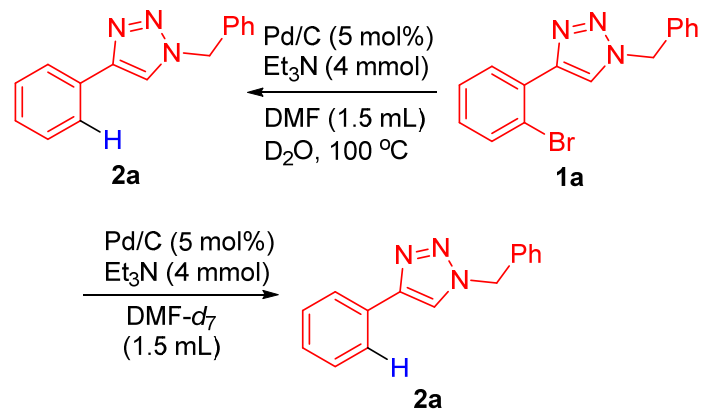

图式 4 控制性实验

Scheme 4 Control experiments

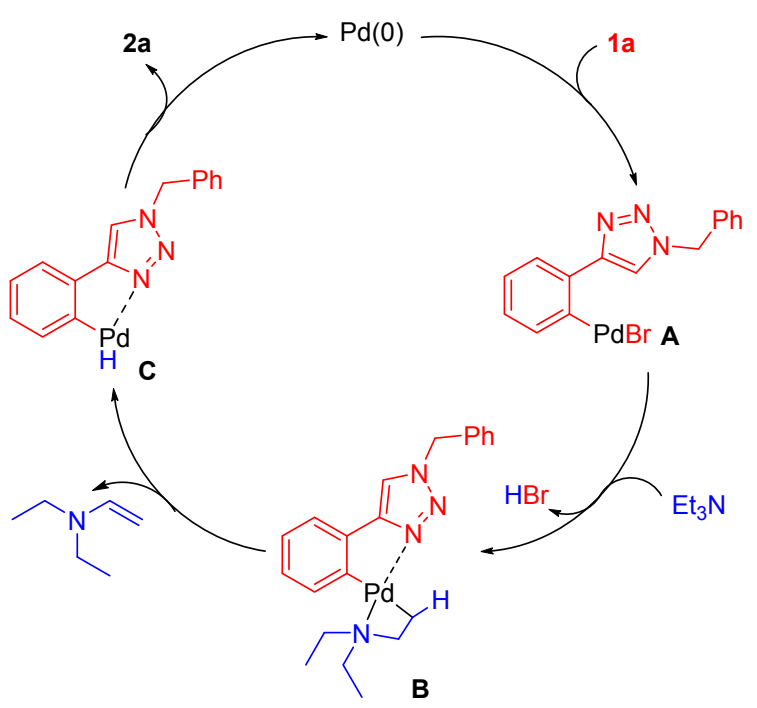

图式 5 可能的反应机理

Scheme 5 Possible reaction mechanism

溶剂为 $\mathrm{CDCl}_{3}$, 内标 TMS; 美国 Thermo 公司 TSQ Quantum Ultra 质谱仪(ESI); 河南省予华仪器有限公司 X-5 型显微熔点仪, 温度计未校正. 所有试剂均为市售 分析纯, 未经进一步纯化.

\section{2 实验方法}

原料邻位三唑取代卤苯由经典的 $\mathrm{CuAAc}$ 反应合成. 邻位三坐取代卤苯的脱卤氢化反应一般步骤: 在装有磁 子的 $15 \mathrm{~mL}$ 刻度试管中加入邻位三唑取代卤苯 $(0.5$ $\mathrm{mmol}) 、 \mathrm{PdCl}_{2}(5 \mathrm{~mol} \%)$ 、三乙胺 $(4 \mathrm{mmol})$ 和 $N, N$-二甲基 甲酰胺(DMF) $(1.5 \mathrm{~mL})$, 然后将试管封闭并置于油浴中 加热反应. 当反应物消失后[用薄层色谱(TLC)跟踪], 将 混合物倒入 $15 \mathrm{~mL}$ 饱和食盐水中, 用二氯甲烷(15 $\mathrm{mL} \times 3$ ) 萃取并合并有机层, 再用无水硫酸镁干燥有机 相, 过滤, 旋干溶剂得粗产物. 所得粗产物通过硅胶柱 层析分离纯化(以石油醚/乙酸乙酯为洗脱剂, $V: V=$ $15: 1 \sim 5: 1$ )得目标产物.

1-苄基-4-苯基-1 $H$-1,2,3-三坐(2a) ${ }^{[13]}$ : 白色固体, 产 率 93\%. m.p. $124 \sim 126{ }^{\circ} \mathrm{C}$ (m.p. $128 \sim 130{ }^{\circ} \mathrm{C}$ ); ${ }^{1} \mathrm{H}$ NMR $\left(\mathrm{CDCl}_{3}, 400 \mathrm{MHz}\right) \delta: 7.81 \sim 7.79(\mathrm{~m}, 2 \mathrm{H}), 7.66(\mathrm{~s}, 1 \mathrm{H})$,
$7.45 \sim 7.36(\mathrm{~m}, 5 \mathrm{H}), 7.35 \sim 7.29(\mathrm{~m}, 3 \mathrm{H}), 5.58(\mathrm{~s}, 2 \mathrm{H}) ;{ }^{13} \mathrm{C}$ NMR $\left(100 \mathrm{MHz}, \mathrm{CDCl}_{3}\right) \delta: 148.16,134.61,130.39$, $129.18,128.82,128.23,128.08,125.75,125.74,119.52$, 54.31; MS (ESI) $m / z: 236[\mathrm{M}+\mathrm{H}]^{+}$.

1-(4-甲基苄基)-4-苯基-1H-1,2,3-三唑(2b): 白色固 体, 产率 94\%, m.p. $91 \sim 92{ }^{\circ} \mathrm{C}$ (lit. ${ }^{[13]}$ m.p. $93 \sim 95{ }^{\circ} \mathrm{C}$ ); ${ }^{1} \mathrm{H}$ NMR $\left(400 \mathrm{MHz}, \mathrm{CDCl}_{3}\right) \delta: 7.80 \sim 7.78(\mathrm{~m}, 2 \mathrm{H}), 7.63$ (s, $1 \mathrm{H}), 7.40 \sim 7.37(\mathrm{~m}, 2 \mathrm{H}), 7.32 \sim 7.28(\mathrm{~m}, 1 \mathrm{H}), 7.23 \sim$ $7.17(\mathrm{~m}, 4 \mathrm{H}), 5.52(\mathrm{~s}, 2 \mathrm{H}), 2.35(\mathrm{~s}, 3 \mathrm{H}) ;{ }^{13} \mathrm{C}$ NMR $(100$ $\left.\mathrm{MHz}, \mathrm{CDCl}_{3}\right) \delta: 148.11,138.75,131.63,130.55,129.81$, $128.79,128.78,128.14,128.13,125.73,125.72,125.71$, $125.69,119.42,54.07,21.16$; MS (ESI) $m / z: 250[\mathrm{M}+\mathrm{H}]^{+}$.

1-(4-叔丁基苄基)-4-苯基- $1 H$-1,2,3-三唑(2c)：白色 固体, 产率 93\%. m.p. 162 164 ${ }^{\circ} \mathrm{C}$ (lit. ${ }^{[14]}$ m.p. 164 $\left.165{ }^{\circ} \mathrm{C}\right) ;{ }^{1} \mathrm{H}$ NMR $\left(400 \mathrm{MHz}, \mathrm{CDCl}_{3}\right) \delta: 7.81 \sim 7.78(\mathrm{~m}$, $2 \mathrm{H}), 7.66(\mathrm{~s}, 1 \mathrm{H}), 7.41 \sim 7.37(\mathrm{~m}, 4 \mathrm{H}), 7.32 \sim 7.28(\mathrm{~m}$, $1 \mathrm{H}), 7.25 \sim 7.24(\mathrm{~m}, 2 \mathrm{H}), 5.53(\mathrm{~s}, 2 \mathrm{H}), 1.31(\mathrm{~s}, 9 \mathrm{H}) ;{ }^{13} \mathrm{C}$ NMR $\left(100 \mathrm{MHz}, \mathrm{CDCl}_{3}\right) \delta: 151.95,148.12,131.64$, $130.57,128.81,128.80,128.79,128.14,127.92,127.91$, $127.90,127.89,126.09,125.71,119.53,53.97,34.67$, 31.27; MS (ESI) $m / z: 292[\mathrm{M}+\mathrm{H}]^{+}$.

4-苯基-1-(4-三氟甲氧基苄基)- $1 H-1,2,3$-三唑(2d): 白色固体, 产率 $90 \%$. m.p. $130 \sim 132{ }^{\circ} \mathrm{C}$ (lit. ${ }^{[13]}$ m.p. $\left.133 \sim 136{ }^{\circ} \mathrm{C}\right){ }^{1} \mathrm{H}$ NMR $\left(400 \mathrm{MHz}, \mathrm{CDCl}_{3}\right) \delta: 7.84 \sim 7.77$ (m, 2H), 7.70 (s, 1H), 7.41 (t, $J=7.4 \mathrm{~Hz}, 2 \mathrm{H}), 7.37 \sim 7.30$ $(\mathrm{m}, 3 \mathrm{H}), 7.24(\mathrm{~d}, J=8.2 \mathrm{~Hz}, 2 \mathrm{H}), 5.58(\mathrm{~s}, 2 \mathrm{H}) ;{ }^{13} \mathrm{C} \mathrm{NMR}$ $\left(100 \mathrm{MHz}, \mathrm{CDCl}_{3}\right) \delta: 149.48,149.46,133.38,130.26$, $129.50,128.86,128.85,128.35,125.75,121.65,121.58$, $121.56,119.48,119.09,53.39$; MS (ESI) $m / z: 320[\mathrm{M}+$ $\mathrm{H}]^{+}$.

1-(4-氟苄基)-4-苯基-1 $H$-1,2,3-三唑(2e)：白色固体, 产率 88\%. m.p. $132 \sim 134{ }^{\circ} \mathrm{C}$ (lit. ${ }^{[13]}$ m.p. $136 \sim 138{ }^{\circ} \mathrm{C}$ ); ${ }^{1} \mathrm{H}$ NMR $\left(400 \mathrm{MHz}, \mathrm{CDCl}_{3}\right) \delta: 7.82(\mathrm{dd}, J=5.2,3.3 \mathrm{~Hz}$, 2H), $7.69(\mathrm{~s}, 1 \mathrm{H}), 7.42(\mathrm{dd}, J=10.3,4.7 \mathrm{~Hz}, 2 \mathrm{H}), 7.34 \sim$ $7.28(\mathrm{~m}, 2 \mathrm{H}), 7.12 \sim 7.06(\mathrm{~m}, 3 \mathrm{H}), 5.55(\mathrm{~s}, 2 \mathrm{H}) ;{ }^{13} \mathrm{C} \mathrm{NMR}$ $\left(100 \mathrm{MHz}, \mathrm{CDCl}_{3}\right) \delta: 164.12,161.65,130.56,130.53$, $130.38,129.99,129.98,129.97,129.90,129.89,128.86$, $128.85,128.84,128.83,128.82,128.81,128.27,125.73$, $125.72,125.70,119.43,116.26,116.05,116.04,53.48 ; \mathrm{MS}$ (ESI) $m / z: 254[\mathrm{M}+\mathrm{H}]^{+}$.

1-(4-氯苄基)-4-苯基-1 $H$-1,2,3-三唑(2f)：白色固体, 产率 $89 \%$. m.p. $152 \sim 154{ }^{\circ} \mathrm{C}$ (lit. ${ }^{[13]}$ m.p. $155 \sim 157{ }^{\circ} \mathrm{C}$ ); ${ }^{1} \mathrm{H}$ NMR $\left(400 \mathrm{MHz}, \mathrm{CDCl}_{3}\right) \delta: 7.80 \sim 7.78(\mathrm{~m}, 2 \mathrm{H}), 7.67$ (s, 1H), $7.41 \sim 7.29(\mathrm{~m}, 5 \mathrm{H}), 7.24 \sim 7.22(\mathrm{~m}, 2 \mathrm{H}), 5.53(\mathrm{~s}$, $2 \mathrm{H}) ;{ }^{13} \mathrm{C}$ NMR $\left(100 \mathrm{MHz}, \mathrm{CDCl}_{3}\right) \delta: 148.34,134.85$, 
$133.19,129.40,129.39,129.38,129.37,129.36,129.35$, $129.34,129.33,128.85,128.30,125.74,119.49,53.49$; MS (ESI) $m / z: 270[\mathrm{M}+\mathrm{H}]^{+}$.

1-(4-硝基苠基)-4-苯基- $1 H$-1,2,3-三唑(2g)：黄色固 体, 产率 85\%. m.p. $155 \sim 157{ }^{\circ} \mathrm{C}$ (lit. ${ }^{[13]}$ m.p. 156 $\left.158{ }^{\circ} \mathrm{C}\right) ;{ }^{1} \mathrm{H}$ NMR $\left(400 \mathrm{MHz}, \mathrm{CDCl}_{3}\right) \delta: 8.26 \sim 8.21(\mathrm{~m}$, 2H), $7.82(\mathrm{dd}, J=5.2,3.3 \mathrm{~Hz}, 2 \mathrm{H}), 7.78(\mathrm{~s}, 1 \mathrm{H}), 7.46 \sim$ $7.40(\mathrm{~m}, 4 \mathrm{H}), 7.36 \sim 7.32(\mathrm{~m}, 1 \mathrm{H}), 5.70(\mathrm{~s}, 2 \mathrm{H}) ;{ }^{13} \mathrm{C}$ NMR $\left(100 \mathrm{MHz}, \mathrm{CDCl}_{3}\right) \delta: 148.61,148.11,141.68,129.94$, $128.93,128.92,128.59,128.58,128.56,125.78,124.33$, 119.82, 77.24, 53.24; MS (ESI) $m / z: 281[\mathrm{M}+\mathrm{H}]^{+}$.

4-苯基-1-(4-三氟甲基苄基)- $1 H$-1,2,3-三坐(2h): 白 色固体, 产率 87\%. m.p. $130 \sim 132{ }^{\circ} \mathrm{C}$ (lit. ${ }^{[15]}$ m.p. $\left.131.9 \sim 132.3{ }^{\circ} \mathrm{C}\right) ;{ }^{1} \mathrm{H} \mathrm{NMR}\left(400 \mathrm{MHz}, \mathrm{CDCl}_{3}\right) \delta: 7.82 \sim$ $7.80(\mathrm{~m}, 2 \mathrm{H}), 7.73(\mathrm{~s}, 1 \mathrm{H}), 7.63(\mathrm{~d}, J=8.1 \mathrm{~Hz}, 2 \mathrm{H}), 7.42 \sim$ $7.38(\mathrm{~m}, 4 \mathrm{H}), 7.35 \sim 7.31(\mathrm{~m}, 1 \mathrm{H}), 5.63(\mathrm{~s}, 2 \mathrm{H}) ;{ }^{13} \mathrm{C} \mathrm{NMR}$ $\left(100 \mathrm{MHz}, \mathrm{CDCl}_{3}\right) \delta: 148.47,138.65,138.64,130.89$, $130.17,128.88,128.87,128.86,128.41,128.21,128.20$, $128.19,128.18,128.17,126.19,126.15,126.11,126.07$, $125.78,125.77,125.76,125.75,125.74,125.13,122.42$, 119.70, 53.56; MS (ESI) m/z: $304[\mathrm{M}+\mathrm{H}]^{+}$.

4-苯基-1-(4-甲酸甲酯苠基)- $1 H$-1,2,3-三唑(2i): 白 色固体, 产率 85\%. m.p. 170 172 ${ }^{\circ} \mathrm{C}$ (lit. ${ }^{[16]}$ m.p. 167 $\left.169{ }^{\circ} \mathrm{C}\right) ;{ }^{1} \mathrm{H}$ NMR $\left(400 \mathrm{MHz}, \mathrm{CDCl}_{3}\right) \delta: 8.04$ (d, $J=8.4$ $\mathrm{Hz}, 2 \mathrm{H}), 7.82 \sim 7.80(\mathrm{~m}, 2 \mathrm{H}), 7.72(\mathrm{~s}, 1 \mathrm{H}), 7.42 \sim 7.38(\mathrm{~m}$, $2 \mathrm{H}), 7.36 \sim 7.30(\mathrm{~m}, 3 \mathrm{H}), 5.63(\mathrm{~s}, 2 \mathrm{H}), 3.91(\mathrm{~s}, 3 \mathrm{H}) ;{ }^{13} \mathrm{C}$ NMR $\left(100 \mathrm{MHz}, \mathrm{CDCl}_{3}\right) \delta: 166.38,148.36,139.49$, $130.62,130.39,130.18,128.87,128.86,128.85,128.37$, $127.81,125.76,119.74,77.25,53.79,52.28$; MS (ESI) $m / z$ : $294[\mathrm{M}+\mathrm{H}]^{+}$.

1-(2-氟苄基)-4-苯基- $1 H$-1,2,3-三唑(2j)：白色固体， 产率 84\%. m.p.92 94 ${ }^{\circ} \mathrm{C}$ (lit. ${ }^{[17]}$ m.p. 91 92 ${ }^{\circ} \mathrm{C}$ ); ${ }^{1} \mathrm{H}$ NMR (400 MHz, $\left.\mathrm{CDCl}_{3}\right) \delta: 7.82 \sim 7.80(\mathrm{~m}, 2 \mathrm{H}), 7.76(\mathrm{~s}$, $1 \mathrm{H}), 7.41 \sim 7.27(\mathrm{~m}, 5 \mathrm{H}), 7.13(\mathrm{dd}, J=16.2,8.0 \mathrm{~Hz}, 2 \mathrm{H})$, $5.62(\mathrm{~s}, 2 \mathrm{H}) ;{ }^{13} \mathrm{C} \mathrm{NMR}\left(100 \mathrm{MHz}, \mathrm{CDCl}_{3}\right) \delta: 161.77$, $159.31,130.96,130.88,130.59,130.56,130.43,128.81$, $128.80,128.21,125.76,125.75,125.74,125.73,124.90$, $124.87,122.06,121.91,119.69,115.94,115.73,47.76$; MS (ESI) $m / z: 254[\mathrm{M}+\mathrm{H}]^{+}$.

1-(2-萗甲基)-4-苯基- $1 H$-1,2,3-三唑(2k)：白色固体， 产率 89\%. m.p. $140 \sim 142{ }^{\circ} \mathrm{C}$ (lit. ${ }^{[18]}$ m.p. $142 \sim 144{ }^{\circ} \mathrm{C}$ ); ${ }^{1} \mathrm{H}$ NMR $\left(400 \mathrm{MHz}, \mathrm{CDCl}_{3}\right) \delta: 7.84 \sim 7.76(\mathrm{~m}, 6 \mathrm{H}), 7.67$ $(\mathrm{s}, 1 \mathrm{H}), 7.52 \sim 7.48(\mathrm{~m}, 2 \mathrm{H}), 7.38 \sim 7.35(\mathrm{~m}, 3 \mathrm{H}), 7.31 \sim$ 7.24(m, 1H), $5.70(\mathrm{~s}, 2 \mathrm{H}) ;{ }^{13} \mathrm{C}$ NMR (100 MHz, $\left.\mathrm{CDCl}_{3}\right) \delta$ : $148.27,133.24,133.21,132.04,130.52,129.22,128.82$,
$128.81,128.19,127.98,127.97,127.95,127.82,127.39$, $126.78,126.76,125.72,125.34,119.65,54.42$; MS (ESI) $m / z: 286[\mathrm{M}+\mathrm{H}]^{+}$.

1-苯乙基-4-苯基- $1 H$-1,2,3-三唑(21): 白色固体，产 率 88\%. m.p. $141 \sim 143{ }^{\circ} \mathrm{C}$ (lit. ${ }^{[16]}$ m.p. $140{ }^{\circ} \mathrm{C}$ ); ${ }^{1} \mathrm{H}$ NMR $\left(400 \mathrm{MHz}, \mathrm{CDCl}_{3}\right) \delta: 7.77 \sim 7.75(\mathrm{~m}, 2 \mathrm{H}), 7.47(\mathrm{~s}, 1 \mathrm{H})$, $7.42 \sim 7.38(\mathrm{~m}, 2 \mathrm{H}), 7.33 \sim 7.23(\mathrm{~m}, 4 \mathrm{H}), 7.14 \sim 7.12(\mathrm{~m}$, $2 \mathrm{H}), 4.62(\mathrm{t}, J=7.2 \mathrm{~Hz}, 2 \mathrm{H}), 3.24(\mathrm{t}, J=7.2 \mathrm{~Hz}, 2 \mathrm{H}) ;{ }^{13} \mathrm{C}$ NMR $\left(100 \mathrm{MHz}, \mathrm{CDCl}_{3}\right) \delta: 147.45,137.06,130.59$, $128.89,128.88,128.87,128.86,128.85,128.84,128.82$, $128.75,128.11,127.15,125.72,125.70,119.97,51.78$, 36.80; MS (ESI) $m / z: 250[\mathrm{M}+\mathrm{H}]^{+}$;

1-戊基-4-苯基- $1 H$-1,2,3-三唑(2m)：白色固体，产率 86\%. m.p. 63 $65{ }^{\circ} \mathrm{C}$ (lit. ${ }^{[19]}$ m.p. $61 \sim 62{ }^{\circ} \mathrm{C}$ ); ${ }^{1} \mathrm{H}$ NMR $\left(400 \mathrm{MHz}, \mathrm{CDCl}_{3}\right) \delta: 7.85 \sim 7.82(\mathrm{~m}, 2 \mathrm{H}), 7.75(\mathrm{~s}, 1 \mathrm{H})$, $7.44 \sim 7.40(\mathrm{~m}, 2 \mathrm{H}), 7.35 \sim 7.30(\mathrm{~m}, 1 \mathrm{H}), 4.38(\mathrm{t}, J=7.2$ $\mathrm{Hz}, 2 \mathrm{H}), 1.98 \sim 1.91(\mathrm{~m}, 2 \mathrm{H}), 1.41 \sim 1.29(\mathrm{~m}, 4 \mathrm{H}), 0.90(\mathrm{t}$, $J=7.0 \mathrm{~Hz}, 3 \mathrm{H}) ;{ }^{13} \mathrm{C} \mathrm{NMR}\left(100 \mathrm{MHz}, \mathrm{CDCl}_{3}\right) \delta: 147.69$, $130.73,128.82,128.07,125.70,125.69,125.68,125.67$, $125.66,119.43,50.43,30.05,28.62,28.61,28.60,22.11$, 13.85; MS (ESI) $m / z: 216[\mathrm{M}+\mathrm{H}]^{+}$

1-环已基-4-苯基-1- $H$-1,2,3-三唑(2n): 产率 67\%. m.p. 103 105 ${ }^{\circ} \mathrm{C}$ (lit. $\left.{ }^{[20]} 104 \sim 105{ }^{\circ} \mathrm{C}\right) ;{ }^{1} \mathrm{H}$ NMR $(400$ $\left.\mathrm{MHz}, \mathrm{CDCl}_{3}\right) \delta: 7.87 \sim 7.84(\mathrm{~m}, 2 \mathrm{H}), 7.79(\mathrm{~s}, 1 \mathrm{H}), 7.48 \sim$ $7.41(\mathrm{~m}, 2 \mathrm{H}), 7.38 \sim 7.32(\mathrm{~m}, 1 \mathrm{H}), 4.52(\mathrm{tt}, J=11.8,3.9$ $\mathrm{Hz}, 1 \mathrm{H}), 2.31 \sim 2.27(\mathrm{~m}, 2 \mathrm{H}), 2.01 \sim 1.94(\mathrm{~m}, 2 \mathrm{H}), 1.87 \sim$ $1.77(\mathrm{~m}, 3 \mathrm{H}), 1.56 \sim 1.45(\mathrm{~m}, 2 \mathrm{H}), 1.39 \sim 1.30(\mathrm{~m}, 1 \mathrm{H})$; ${ }^{13} \mathrm{C}$ NMR $\left(100 \mathrm{MHz}, \mathrm{CDCl}_{3}\right) \delta: 147.22,130.72,128.82$, 128.81, 128.05, 125.68, 117.34, 60.24, 33.62, 25.20, 25.16; MS (ESI) $m / z: 228[\mathrm{M}+\mathrm{H}]^{+}$.

1-苄基-4-对甲基苯基-1H-1,2,3-三唑(2o): 黄色固 体, 产率 92\%. m.p. 155 157 ${ }^{\circ} \mathrm{C}$ (lit. ${ }^{[13]} 152 \sim 154{ }^{\circ} \mathrm{C}$ ); ${ }^{1} \mathrm{H}$ NMR $\left(400 \mathrm{MHz}, \mathrm{CDCl}_{3}\right) \delta: 7.69 \sim 7.67$ (m, 2H), 7.62 $(\mathrm{s}, 1 \mathrm{H}), 7.40 \sim 7.35(\mathrm{~m}, 3 \mathrm{H}), 7.31 \sim 7.29(\mathrm{~m}, 2 \mathrm{H}), 7.20(\mathrm{~d}$, $J=7.9 \mathrm{~Hz}, 2 \mathrm{H}), 5.55(\mathrm{~s}, 2 \mathrm{H}), 2.36(\mathrm{~s}, 3 \mathrm{H}) ;{ }^{13} \mathrm{C}$ NMR $(100$ $\left.\mathrm{MHz}, \mathrm{CDCl}_{3}\right) \delta: 148.29,138.03,134.73,129.50,129.14$, $128.76,128.08,128.07,128.06,128.05,127.68,125.61$, 119.18, 77.25, 54.21, 21.29; MS (ESI) m/z: $250[\mathrm{M}+\mathrm{H}]^{+}$.

1-芐基-4-(4-氟苯基)- $1 H$-1,2,3-三唑(2p): 白色固体, 产率 78\%. m.p. $112 \sim 114{ }^{\circ} \mathrm{C}$ (lit. $\left.{ }^{[21]} 113 \sim 115{ }^{\circ} \mathrm{C}\right) ;{ }^{1} \mathrm{H}$ NMR $\left(400 \mathrm{MHz}, \mathrm{CDCl}_{3}\right) \delta: 7.83 \sim 7.74(\mathrm{~m}, 2 \mathrm{H}), 7.66(\mathrm{~s}$, $1 \mathrm{H}), 7.44 \sim 7.37(\mathrm{~m}, 3 \mathrm{H}), 7.36 \sim 7.29(\mathrm{~m}, 2 \mathrm{H}), 7.13 \sim 7.08$ $(\mathrm{m}, 2 \mathrm{H}), 5.58(\mathrm{~s}, 2 \mathrm{H}) ;{ }^{13} \mathrm{C} \mathrm{NMR}\left(100 \mathrm{MHz}, \mathrm{CDCl}_{3}\right) \delta$ : $163.90,161.44,147.32,134.57,129.21,129.20,129.19$, $129.18,129.16,128.85,128.09,127.50,127.43,126.72$, 
119.31, 115.90, 115.68, 54.30; MS (ESI) m/z: $254[\mathrm{M}+$ $\mathrm{H}]^{+}$.

1-芐基-4-(4-三氟甲氧基苯基)- $1 H$-1,2,3-三唑(2q) ${ }^{[22]}$ : 产率 $83 \%$. 粘稠液体. ${ }^{1} \mathrm{H} \mathrm{NMR}\left(400 \mathrm{MHz}, \mathrm{CDCl}_{3}\right) \delta$ : $7.86 \sim 7.80(\mathrm{~m}, 2 \mathrm{H}), 7.67(\mathrm{~s}, 1 \mathrm{H}), 7.42 \sim 7.35(\mathrm{~m}, 3 \mathrm{H})$, $7.34 \sim 7.30(\mathrm{~m}, 2 \mathrm{H}), 7.24(\mathrm{dd}, J=8.8,0.8 \mathrm{~Hz}, 2 \mathrm{H}), 5.57$ (s, $2 \mathrm{H}) ;{ }^{13} \mathrm{C} \mathrm{NMR}\left(100 \mathrm{MHz}, \mathrm{CDCl}_{3}\right) \delta: 148.98,134.49$, $129.31,129.21,128.89$, 128.11, 128.10, 128.08, 127.09, 121.33, 119.69, 119.18, 77.24, 54.33; MS (ESI) $m / z: 320$ $[\mathrm{M}+\mathrm{H}]^{+}$.

1-苯基-4-对甲基苯基- $1 H-1,2,3$-三唑(3a): 白色固 体, 产率 90\%. m.p. $160 \sim 162{ }^{\circ} \mathrm{C}$ (lit. ${ }^{[23]} 160 \sim 162{ }^{\circ} \mathrm{C}$ ); ${ }^{1} \mathrm{H}$ NMR $\left(400 \mathrm{MHz}, \mathrm{CDCl}_{3}\right) \delta: 8.15(\mathrm{~s}, 1 \mathrm{H}), 7.79(\mathrm{t}, J=7.6$ $\mathrm{Hz}, 4 \mathrm{H}), 7.54(\mathrm{dd}, J=14.3,6.8 \mathrm{~Hz}, 2 \mathrm{H}), 7.47 \sim 7.39(\mathrm{~m}$, $1 \mathrm{H}), 7.30 \sim 7.24(\mathrm{~m}, 2 \mathrm{H}), 2.39(\mathrm{~s}, 3 \mathrm{H}) ;{ }^{13} \mathrm{C}$ NMR $(100$ $\left.\mathrm{MHz}, \mathrm{CDCl}_{3}\right) \delta: 148.49,138.31,137.12,129.79,129.60$, $128.69,127.43,125.77,120.58,120.49,117.26$, 99.98, 21.33; MS (ESI) $m / z: 236[\mathrm{M}+\mathrm{H}]^{+}$.

4-(4-甲氧基苯基)-1-苯基- $1 H$-1,2,3-三唑(3b): 白色 固体，产率 $89 \%$. m.p. $149 \sim 152 \quad{ }^{\circ} \mathrm{C} \quad\left(\right.$ lit. $^{[24]} 150 \sim$ $\left.152{ }^{\circ} \mathrm{C}\right) ;{ }^{1} \mathrm{H} \mathrm{NMR}\left(400 \mathrm{MHz}, \mathrm{CDCl}_{3}\right) \delta: 8.11$ (s, 1H), 7.84 $(\mathrm{d}, J=8.7 \mathrm{~Hz}, 2 \mathrm{H}), 7.79$ (d, $J=7.8 \mathrm{~Hz}, 2 \mathrm{H}), 7.54$ (t, $J=7.8$ $\mathrm{Hz}, 2 \mathrm{H}), 7.45$ (t, $J=7.4 \mathrm{~Hz}, 1 \mathrm{H}), 6.99$ (d, $J=8.7 \mathrm{~Hz}, 2 \mathrm{H})$, $3.86(\mathrm{~s}, 3 \mathrm{H}) ;{ }^{13} \mathrm{C} \mathrm{NMR}\left(100 \mathrm{MHz}, \mathrm{CDCl}_{3}\right) \delta: 159.83$, $129.76,128.68,127.19,122.93,120.50,114.35,55.36$, 29.71; MS (ESI) $m / z: 252[\mathrm{M}+\mathrm{H}]^{+}$.

4-丁基-1-苯基- $1 H-1,2,3$-三唑(3c $)^{[24]}$ : 浅黄色透明液 体, 产率 84\%. ${ }^{1} \mathrm{H}$ NMR (400 $\left.\mathrm{MHz}, \mathrm{CDCl}_{3}\right) \delta: 7.74 \sim 7.71$ $(\mathrm{m}, 3 \mathrm{H}), 7.53 \sim 7.49(\mathrm{~m}, 2 \mathrm{H}), 7.41(\mathrm{t}, J=7.4 \mathrm{~Hz}, 1 \mathrm{H}), 2.81$ $(\mathrm{t}, J=7.6 \mathrm{~Hz}, 2 \mathrm{H}), 1.77 \sim 1.69(\mathrm{~m}, 2 \mathrm{H}), 1.48 \sim 1.39(\mathrm{~m}$, 2H), 0.96 (t, $J=7.4 \mathrm{~Hz}, 3 \mathrm{H}) ;{ }^{13} \mathrm{C} \mathrm{NMR}\left(100 \mathrm{MHz}, \mathrm{CDCl}_{3}\right)$ $\delta: 149.16,137.31,129.67,129.65,128.41,120.41,31.50$, 25.35, 22.32, 13.82; MS (ESI) $m / z: 202[\mathrm{M}+\mathrm{H}]^{+}$.

辅助材料(Supporting Information) 化合物 $\mathbf{2 a} \sim \mathbf{2 q}$ 和 $3 \mathbf{a} \sim 3 \mathbf{c}$ 的核磁共振氢谱和碳谱. 这些材料可以免费从 本刊网站(http: //sioc-journal.cn/)上下载.

\section{References}

[1] (a) Mitoma, Y.; Nagashima, S.; Simion C.; Simion A. M.; Yamada, T.; Mimura K.; Ishimoto, K.; Tashiro, M. Environ. Sci. Technol.
2001, 35, 4145.

(b) Mitoma, Y.; Uda, T.; Egashira, N.; Simion, C.; Tashiro, H.; Tashiro, M.; Fan, X. Environ. Sci. Technol. 2004, 38, 1216.

(c) Mitoma, Y.; Tasaka, M.; Takase, M.; Masuda, T.; Tashiro, H.; Egashira, N.; Oki, T. Environ. Sci. Technol. 2006, 40, 1849.

(d) Mitoma, Y.; Kakeda, M.; Simion, A. M.; Egashira, N.; Simion, C. Environ. Sci. Technol. 2009, 43, 15, 5952.

[2] Alonso, F.; Beletskaya, I. P.; Yus M. Chem. Rev. 2002, 102, 4009.

[3] (a) Guram, A. S.; Rennels, R. A.; Buchwald, S. L. Angew. Chem., Int. Ed. Engl. 1995, 34, 1348.

(b) Marcoux, J.-F.; Wagaw, S.; Buchwald, S. L. J. Org. Chem. 1997, 62, 1568 .

(c) Wolfe, J. P.; Buchwald, S. L. J. Org. Chem. 2000, 65, 1144.

[4] (a) Jones, J. R.; William, J. S.; Lockley, S. L.; Stewart, P. Tetrahedron Lett. 2001, 42, 331.

(b) Uozumi, Y.; Nakao, R.; Rhee, H. Org. Lett. 2005, 7, 163.

[5] (a) Rodriguez, J. G.; Lafuente, A. Tetrahedron Lett. 2002, 43, 9645. (b) Cellier, P. P.; Spindler, J. F.; Taillefera, M.; Cristaua, H. J. Tetrahedron Lett. 2003, 44, 7191.

[6] Zawisza, A. M.; Muzart, J. Tetrahedron Lett. 2007, 48, 6748. (DMF)

[7] (a) Viciu, M. S.; Grasa, G. A.; Nolan, S. P. Organometallics 2001, $20,3607$.

(b) Navarro, O.; Kaur, H.; Nolan, S. P. J. Org. Chem. 2004, 69, 3173.

[8] Pyo, A.; Kin, S.; Kumar, M. R.; Byeun, A.; Eom, M. S.; Han, M. S.; Lee, S. Tetrahedron Lett. 2013, 54, 5207.

[9] Yamamoto, Y.; Nogi, K.; Yorimitsu, H.; Osuka, A. ChemistrySelect 2017, 2, 1723.

[10] Bhattacharjya, A.; Klumphu, P.; Lipshutz, B. H. Org. Lett. 2015, 17, 1122.

[11] Qiu, H.; Zhou, P.; Liu, W.; Zhang, J.; Chen, B. ChemistrySelect 2020, 5, 2935.

[12] (a) Zou, B.; Jiang, H.; Wang, Z. Eur. J. Org. Chem. 2007, 4600.

(b) Zou, B.; Jiang, H. Chin. J. Chem. 2008, 26, 1309.

(c) Roy, S. K.; Tiwari, A.; Saleem, M.; Jana, C. K. Chem. Commun. 2018, 54, 14081 .

(d) Zhang, D.; Khan, R.; Yang, F.; Zhang, X.; Shen, G.; Gao, Y.; Fan, R.; Sun, W.; Fan, B. Eur. J. Org. Chem. 2018, 3464.

[13] Zhang, Z.; Song, P.; Zhou, J.; Chen, Y.; Lin, B.; Li, Y. Ind. Eng. Chem. Res. 2016, 55, 12301.

[14] Özçubukçu, S.; Ozkal, E.; Jimeno, C.; Pericàs, M. A. Org. Lett. 2009, 11, 4680 .

[15] Asano, K.; Matsubara, S. Org. Lett. 2010, 12, 4988.

[16] Shin, J.; Lim, Y.; Lee, K. J. Org. Chem. 2012, 77, 4117.

[17] Adenot, A.; Landstrom, E. B.; Gallou, F.; Lipshutz, B. H. Green Chem. 2017, 19, 2506.

[18] Ötvös, S. B.; Hatoss, G.; Georgiádes, Á.; Kovács, S.; Mándity, I. M.; Novák, Z.; Fülöp, F. RSC Adv. 2014, 4, 46666.

[19] Chen, Z.; Yan, Q.; Yi, H.; Liu, Z.; Lei, A.; Zhang, Y. Chem.-Eur. J. 2014, 20, 13692

[20] Asano, K.; Matsubara, S. Org. Lett. 2010, 12, 4988.

[21] Park, I. S.; Kwon, M. S.; Kim, Y.; Lee, J. S.; Park, J. Org. Lett. 2008, 10, 497.

[22] Yamada, Y.M.A.; Ohno, A.; Sato, T.; Uozumi, Y. Chem.-Eur. J. 2015, 21, 17269.

[23] Ramachary, D.B., Shashank, A.B. and Karthik, S. Angew. Chem., Int. Ed. 2014, 53, 10420.

[24] Kafle, A.; Handy, S. T. Tetrahedron 2017, 73, 7024. 